\title{
Le détective biblique : Daniel et le débat sur une préhistoire du roman policier
}

\section{Rebecca JOSEPHY}

Le roman policier - ainsi que nous le disent les experts - est un genre littéraire moderne naissant au dix-neuvième siècle avec la révolution industrielle, d'importants progrès scientifiques et des changements dans la structure des sociétés européennes et nord-américaines. La population des métropoles, en effet, grandit. Paris devient cette grande « capitale du dix-neuvième siècle », à laquelle se réfère Walter Benjamin, et de ses profondeurs jaillissent les interminables grondements des bas-fonds. Prostituées, vices, misère, alcool... tout contribue à rendre les allées, les bouges et les coins ombragés les lieux d'une bourdonnante activité marginale. La préfecture de police symboliquement créée par le décret napoléonien du 17 février 1800 - se retrouve impliquée dans des enquêtes toujours plus nombreuses. Le dix-neuvième siècle, comme l'indique bien Dominique Kalifa, « fut obsédé par la question du crime. L’observer, le punir, le résorber, constituèrent des préoccupations majeures, que justifiait aux yeux des contemporains le sentiment d'une criminalité chaque jour plus envahissante » (9).

Tout ceci est exact, tout comme il est juste de souligner l'importance de la presse au dixneuvième siècle et la création d'un véritable commerce littéraire et journalistique du crime. En 1836, Lacenaire, poète éloquent et meurtrier, nommé « le monstre de la bourgeoisie », devient un phénomène de presse lorsque son visage angélique et ses vers sentimentaux écrits pendant son incarcération deviennent difficilement conciliables avec l'image d'un criminel sans cœur. En 1869, l'affaire Troppmann captive l'attention de la population en raison, au contraire, de son extrême brutalité. Jeune Alsacien, il assassine les Kinks, père et fils ainé, afin de récupérer l'argent de la famille. Incapable d'accéder aux biens convoités, il finit par étrangler et poignarder les six autres membres de la famille au cours d'une nuit sanglante, connue sous le nom du massacre de Pantin. Du côté de la Grande Bretagne, les crimes se multiplient également et atteignent un statut mythique dans le fameux quartier White Chapel où Jack l'éventreur tue et mutile des prostituées durant une période de plusieurs années.

Il faut aussi ne pas négliger la dynamique qui s'impose entre criminel et enquêteur. La personnalité des criminels fait peur et leurs actes exigent des réponses. Vidocq, ce petit escroc duquel s'inspirent maintes figures littéraires, devient informateur et finit par être le chef de la 
Sûreté Nationale. En 1828, il publiera ses mémoires à l'aide de deux polygraphes et rendra dès lors publiques les pratiques policières. Dans les années qui suivent, d'autres hommes prendront la tête de cette organisation anti-crime et, comme leur prédécesseur, ils prendront également la plume : Philippe Auguste Cattelain, Antoine Claude, Gustave Macé, Marie-François Goron, Louis Canler, etc. Leurs mémoires et récits contribueront à mettre en lumière une culture où l'espionnage, les opérations clandestines, les informateurs et les contre-interrogatoires règnent. Il ne s'agit pas, en effet, uniquement de crimes sensationnels, mais d'une criminalité qui devient sophistiquée, qui se cache plus facilement et qui exige plus de finesse de la part de la préfecture.

C'est un genre littéraire qui est donc fortement enraciné dans un temps et lieu précis et qui ne peut, selon Régis Messac, avoir trouvé

la complexité et la variété qui lui sont nécessaires que dans une société moderne, où la population étant concentrée dans des espaces relativement restreints, chacun s'observe chaque jour de plus près, où le poursuivi met chaque jour plus d'ingéniosité à se cacher, et le poursuivant plus de subtilité à découvrir les cachettes, où les ruses perfectionnées des voleurs provoquent le perfectionnement croissant des méthodes policières. (104)

Et pourtant, pour autant que le roman policier fleurit au dix-neuvième siècle et reflète une réalité bien moderne certainement nourrie, voire peut-être déclenchée par de profondes évolutions historiques - ce qui expliquerait sa domination aux États-Unis, en Angleterre et en France, où ces réalités étaient les plus tangibles - il existe bien un exemple lointain et biblique de détective avant la lettre.

Daniel, ce sage de la Bible qui démêle des mystères, des puzzles et des casse-tête, exemplifie les qualités les plus caractéristiques du détective. Il recourt à la raison, utilise des situations difficiles à son avantage, lit les personnes au-delà leurs apparences et se fie à l'évidence afin de déterminer leur innocence ou culpabilité. Dans le premier chapitre, on apprend que Daniel et ses compagnons sont «instruits en toute sagesse, savants en science et subtils en savoir » (Dn I, 4). Dans le cinquième chapitre, il déchiffre une inscription cryptique et l'on découvre qu'il se trouve en lui « un esprit extraordinaire, connaissance, intelligence, art d'interpréter les songes, de résoudre les énigmes et de défaire les nœuds » (Dn V, 12). Dans le chapitre deutérocanonique de «Bel et le serpent», il résout l'énigme d'un vol en analysant des empreintes et dans «Susanne et les vieillards », il piège deux coupables en menant un contre-interrogatoire digne des techniques policières les plus éprouvées. Plusieurs épisodes de ce livre biblique contiennent, en effet, des scènes ou éléments archétypaux du roman policier moderne - le mystère de la chambre close, 
l'inscription cryptique ou codée, tactiques de contre-interrogatoire. Ainsi, dans les premiers travaux critiques sur le genre du roman policier (Dorothy Sayers, Edward Wrong, Régis Messac) ces éléments sont signalés, mais de façon assez superficielle. Les travaux plus récents ne sont pas venus combler ce manque. En effet, les considérations sur Daniel dépassent rarement quelques phrases, comme si toute exploration plus poussée concernant les ancêtres du roman policier constituait un terrain dangereux, compliqué, voire tabou. Pourquoi cette histoire souterraine du roman policier moderne est-elle donc restée largement inexplorée jusqu'à date ?

La réponse réside certainement dans cette association puissante entre le roman policier et la modernité, ainsi que l'explique Julian Symons :

Les historiens se divisent entre ceux qui soutiennent qu'il n'y a pu y avoir de romans policiers avant qu'existent des forces de police et de recherche organisées, et ceux qui découvrent des exemples de déduction rationnelle aussi bien dans la Bible que chez Voltaire. Pour le premier groupe, le roman policier commence avec Edgar Allan Poe, pour le second, il a ses racines dans les débuts mêmes de l'histoire connue. $(23)^{1}$

Le paragraphe résume bien le débat, mais pose les enjeux de manière conflictuelle, comme si toute recherche concernant une préhistoire du roman policier négligeait implicitement la spécificité des romans policiers modernes, comme s'il était impossible de reconnaître à la fois la modernité du genre, son explosion à la fin du dix-neuvième siècle et les aspects du genre comme l'enquête, le mystère, les énigmes et les puzzles qui apparaissent dans des récits qui précèdent sa naissance.

Ce paragraphe de Symons perpétue également des approximations au niveau de la langue, car malgré la terminologie française, les romans policiers classiques reposent non pas sur la figure d'un héros policier, mais sur celle d'un détective. La distinction nous paraît essentielle, car si les détectives modernes - les Sherlock Holmes, les Miss. Marple, les Poirot, les Rouletabille et autre - se servent souvent des tactiques de la police, ces derniers sont loin d'être contraints à ses procédures $^{2}$. Le roman policier joue, en fait, sur l'opposition entre police et détective ; entre l'institution incompétente et impuissante d'une part, et l'individu exceptionnel, excentrique, quasidivin, de l'autre. Comment donc expliquer la réticence à attribuer l'étiquette de détective ou à la limite de proto-détective à un personnage antique qui déchiffre des énigmes ? S'agit-il simplement d'une question sémantique - à savoir, un argument basé dans une logique de puriste selon laquelle des éléments du roman policier ne peuvent exister avant que le genre ne soit défini ?

Pour autant que cet argument soit circulaire, c'est bien celui-ci qu'on rencontre le plus souvent. Dans son étude « Murder for Pleasure », Howard Haycraft l'exprime ainsi : « Les paroles 
les plus définitives et les plus autorisés sur ce sujet ont été prononcées par le bibliophile Georges Bates : 'La raison du silence de Chaucer à propos des avions était qu'il n'en avait jamais vu aucun. Vous ne pouvez pas écrire sur des policiers avant que la police n'existe' » $(162)^{3}$. De nouveau le choix de langue est significatif, car comme Symons qui a employé le mot «police » plutôt que «détective » afin d'ancrer le genre littéraire du roman policier fermement dans une réalité moderne, Haycraft adopte lui-aussi cette même terminologie. ${ }^{4}$ Dans une étude qui recourt presque exclusivement au mot «détective » privilégié par la critique anglophone, il passe abruptement au mot « police ».

À cet argument axé sur une distinction police/détective, s'ajoute, nous semble-t-il, une raison plus profonde à l'absence d'étude sur Daniel. Celle-ci porte sur le statut historique du roman policier comme genre inférieur. Le roman policier, nous le savons bien, n'a jamais eu bonne presse et a été longtemps accusé d'être une sous-littérature, un passe-temps frivole, une entreprise, en somme, anecdotique. Dans ses articles polémiques parus dans le New Yorker d'octobre 1944 et de juillet 1945, « Pourquoi les gens lisent-ils des romans policiers? » et « Que nous importe le meurtre de Roger Ackroyd $»^{5}$, Edmund Wilson a soutenu que depuis la fin du dix-neuvième siècle, le roman policier n'a « fait que décliner » (83) et que cette littérature n'est pas faite pour être lue, mais plutôt pour être parcourue «à toute vitesse pour voir comment se résout le problème » (86). On ne peut, continue-t-il «s'intéresser aux personnages parce qu'ils n'ont jamais d'existence propre, même à plat, en deux dimensions ... » (86). Raymond Chandler, champion et écrivain dans le courant du Hard boiled, va encore plus loin en critiquant toute la littérature concernée principalement avec des indices, y compris celle de Conan Doyle, la blâmant pour ses intrigues clichées, peu crédibles et ne plaisant qu'aux gouts des masses (The Simple Art of Murder).

En ce qui concerne une préhistoire du roman policier, cette infériorité supposée ou ressentie ne cesse d'entrer en ligne de compte et s'insinue dans le débat, comme le laisse entendre Leroy Lad Panek : «regarder la lignée remontant à Moïse illustre aussi l'infériorité ressentie consciemment par les écrivains de romans policiers concernant leur travail $»(7)^{6}$, ou encore comme l'exprime Yves Reuter : « il semble [...] que la recherche de sources tiennent plus à une volonté de valoriser le genre par de 'grands' ancêtres qu'à une quelconque réalité » (11). Nous voici ainsi au cœur du problème : une distinction qui repose sur une question de statut, comme si toute recherche sur les ancêtres du roman policier entendaient implicitement une transgression de la frontière entre écrivains artistes et écrivains tâcherons de la littérature de masse, comme si le 
vitriol porté contre les études du roman policier avait mené paradoxalement à une forme de revendication de son statut comme paralittérature, et une vigilance parmi ses chercheurs contre ceux qui pourraient mettre cette marginalité en danger.

Dans le cas tout particulier du Livre de Daniel, cette distinction n'est cependant pas opérationnelle. Il s'agit, en effet, d'un personnage biblique qui a connu une grande popularité dès son origine. La découverte des douze chapitres canoniques du Livre de Daniel parmi les manuscrits de la Mer Morte nous montre que le livre faisait partie du canon hébreu environ quarante ans après sa compilation et que Daniel en tant que un personnage littéraire était donc lu, étudié et admiré peu de temps après sa rédaction ${ }^{7}$. De façon plus générale, la Bible est, nous le savons bien, le premier livre à être imprimé et diffusé en grande circulation, le premier à dépasser les milliards de ventes, suivi, d'ailleurs - et cela n'est sans doute pas anodin - par l'œuvre d'Agatha Christie ${ }^{8}$. Audelà de la pratique religieuse, les textes bibliques, comme les romans policiers modernes, ont largement infusé la culture de masse.

Néanmoins, la réticence liée au sujet des ancêtres bibliques du roman policier persiste et limite les interrogations sur ce genre littéraire. Les épisodes où Daniel agit en tant que détective ouvrent le champ et nous invite à mettre en question les rapports entre le roman policier, la modernité et l'avènement d'un rationalisme utilitariste. Dans tous les récits du Livre de Daniel où Daniel résout une énigme, on retrouve, en fait, une pensée religieuse qui cohabite avec une pensée rationnelle. Dans le cinquième chapitre du livre, Daniel se réfère de nombreuses fois à son Dieu, il accepte sans l'interroger l'arrivée d'une main autonome. Cependant, le héros hébreu écarte des explications surnaturelles, utilise sa raison pour déchiffrer le cryptogramme et mène une enquête sur les rois babyloniens. Dans l'épisode de «Susanne et les vieillards », Dieu révèle à Daniel l'innocence de la jeune femme (Dn XIII, 45-47) ${ }^{9}$ et pourtant, il mène néanmoins le contreinterrogatoire qui prouve la culpabilité des vieillards. De même, dans « Bel et le serpent », il croit fermement en son Dieu « vivant qui a fait le ciel et la terre et qui a puissance sur toute chair » (Dn XIV, 4) en même temps qu'il se moque ouvertement des croyances idolâtres des Babyloniens. L'apparition de récits axés sur des puzzles et des énigmes dans le texte biblique semble donc tenir d'une coexistence entre le naturel et le surnaturel que d'un rejet total de ce dernier.

Ces éléments qui nous offrent un aperçu sur la genèse des récits bibliques où Daniel agit en tant que proto-détective, ne sont pas sans lien avec le roman policier moderne. Les tous premiers écrivains de roman policier comme Poe ou Hoffman étaient d'abord des écrivains connus pour 
leurs affinités avec le fantastique. Jean-Claude Vareille avait déjà relevé ces convergences entre le roman gothique, le roman populaire et le roman policier naissant: « le roman gothique anglais, dès la fin du XVIIIe siècle, et le roman feuilleton ou populaire, présente déjà un caractère essentiel au roman policier [...]. Dans le roman noir ou le roman populaire le mystère est partout » (42).

Une analyse détaillée du roman policier montre, en fait, que non seulement la transition entre naturel et surnaturel est beaucoup plus fluide que les théories traditionnelles la présentent, mais aussi que le roman policier comporte des aspects profondément théologiques. Nombre de détectives modernes comme Guillaume de Baskerville, Father Brown, Rabbi Small, sont des membres du clergé. Loin d'être des curiosités, ces personnages révèlent un aspect inhérent à cette littérature : le détective, comme le prophète, est capable de voir ce que personne d'autre n'est capable de voir et d'aller au-delà les apparences. Ces êtres quasi-divins qui partent à la rencontre de chaque nouveau mystère, les yeux brillants, presque plongés dans un état de transe, finissent par miraculeusement délivrer les lecteurs de leurs incertitudes, subjugués par le pouvoir de ces modernes prophètes de dire la vérité.

Le moment ultime où le détective perce le code, défait le nœud, résout l'énigme reprend ainsi le même processus que celui au cœur de la révélation. Énigme après énigme, le lecteur suivant à la trace le détective se retrouve lui-même - au moins en partie - dans cette position d'herméneute. Le contraire serait à la fois impensable - quel lecteur du roman policier se désintéresse-t-il de la résolution de l'énigme ? - et insupportable, comme l'indique Léon Chestov : «Nous vivons entourés d'une multitude infinie de mystères. Mais, si énigmatiques que soient les mystères qui entourent l'être, ce qu'il y a de plus énigmatique et de plus inquiétant, c'est que le mystère existe en général, c'est que nous soyons en quelque sorte définitivement et pour toujours retranchés des sources et des commencements de la vie » (73). Addictif et itératif, le roman policier remplit ainsi cette fonction essentielle qui est aussi celle des religions, de répondre au mystère, de dissiper les inquiétudes et de révéler une vérité cachée.

Le Livre de Daniel nous oblige, en effet, à considérer le roman policier sous un autre éclairage, à repenser ce que l'on qualifie habituellement de «moderne » ou de «scientifique ». Ces récits détectives, tout comme les récits théologiques, mettent en leur cœur des mystères et des questions, les prennent en charge pour le lecteur, les font disparaître pour finalement laisser place à une épiphanie. II ne s'agit pas que de fascination pour la science, il ne s'agit pas uniquement de 
justice, il s'agit surtout de se rassurer et de fournir une réponse : qui croire, qui vénérer, qui écouter et à qui se fier.

\section{Bibliographie}

Chandler, Raymond. The Simple Art of Murder. Boston : Houghton Mifflin / The Riverside P Cambridge, 1950.

Chestov, Léon. Athènes et Jérusalem. Paris : Bruit du temps, 2011.

Eisenzweig, Uri, dir. Autopsies du roman policier. Paris : Union Générale d'éditions, 1983.

Haycraft, Howard. « Murder for pleasure ». The Art of the Mystery Story. Dir. Howard Haycraft. New York : Carroll \& Graf, 1983.

Kalifa, Dominique. Crime et culture au XIXe siècle. Paris : Perrin, 2005.

Messac, Régis. Le «Detective Novel» et l'influence de la pensée scientifique. Dir. Jean-Luc Buard, Hélène Chantemerle, Antoine Lonnet et Olivier Messac. Paris : Encrage, 2011. [1929]

Panek, Leroy Lad. An Introduction to the Detective Story. Bowling Green, Ohio : State U Popular P, 1987.

Reuter, Yves. Le roman policier. Paris : Colin, 2009.

Symons, Julian. Bloody Murder. From the Detective Story to the Crime Novel : A History. New York : Penguin, 1974.

Vareille, Jean-Claude. L'homme masqué : le justicier et le détective. Lyon : PU de Lyon.

Wilson, Edmund. «Pourquoi les gens lisent-ils des romans policiers ? / «Que nous importe le meurtre de Roger Ackroyd ». Autopsies du roman policier. Dir. Uri Eisenzweig. Tr. Claude Gilbert. Paris : Union Générale d'éditions, 1983.

\section{NOTES}

${ }^{1}$ Ma traduction de l'original : « Historians of the detective story are divided between those who say that there could be no detective stories until organized police and detective forces existed, and those who find examples of rational deduction in sources as various as the Bible and Voltaire, and suggest that these were early puzzles in detection. For the first group the detective story begins with Edgar Allan Poe, for the second its roots are in the beginnings of recorded history. »

${ }^{2} \mathrm{C}$ 'est la raison pour laquelle on retrouve autant de romans policiers historiques qui ont pour cadre des lieux qui antidatent la création de la police telle l'Égypte ancienne, ou encore, comme dans le cas du Nom de la rose, le Moyen Âge tardif. 


\footnotetext{
${ }^{3}$ Ma traduction de l'original : "The best and final word on the matter has been said by the English bibliophile George Bates: 'The cause of Chaucer's silence on the subject of airplanes was because he had never seen one. You cannot write about policemen before policemen exist to be written of.' »

${ }^{4} \mathrm{Si}$ le mot «détective » n'a pas existé avant le dix-neuvième siècle et qu'à son origine il était bien associé à une branche de Scotland Yard et donc à la police, dans la littérature, ce personnage se définit simplement par sa capacité exceptionnelle à résoudre des énigmes. Sur l'origine du mot « détective», voir Uri Eisenzweig, dir. Autopsies du roman policier (9).

${ }^{5}$ Ces articles ont les titres originels suivants : «Why do People Read Detective Stories ? » et « Who Cares Who Killed Roger Ackroyd ».

${ }^{6}$ Ma traduction de l'original : « Looking for a lineage stretching back to Moses also illustrates the conscious inferiority felt by detective writers about their work $»$.

${ }^{7}$ En dépit du fait que les récits qui lui sont consacrés font maintenant parmi des Kétuvim (Écritures) et non des Néviims (Prophètes) dans la Bible hébraïque, à l'époque de sa rédaction Daniel était considéré comme un prophète.

8 Voir la présentation de l'exposition sur Agatha Christie à Montréal du 8 décembre au 17 avril 2016: http://www.pacmusee.qc.ca/en/exhibitions/agatha-christie-and-archaeology

9 «[...] Dieu éveilla l'esprit saint d'un jeune enfant nommé Daniel. Il cria à haute voix: 'Pour moi, je suis pur du sang de cette femme!'»
} 